\section{Nanomaterials arising amid antibiotic resistance}

\author{
Weiwei Gao 10 and Liangfang Zhang
}

The rapid emergence of antibiotic resistance is recognized as a major public health threat. Nanomaterials have risen to tackle this problem through either improving the potency of existing antibiotics or generating entirely new antibacterial mechanisms.

Refers to Makabenta, J. M. V. et al. Nanomaterial-based therapeutics for antibiotic-resistant bacterial infections. Nat. Rev. Microbiol. https://doi.org/10.1038/s41579-41020-40420-41571 (2020) | Linklater, D. P. et al. Mechano-bactericidal actions of nanostructured surfaces. Nat. Rev. Microbiol. https://doi.org/10.1038/s41579-41020-40414-z (2020).

Our awareness of infectious diseases has recently been heightened by the current outbreak of coronavirus. The crisis is unprecedented and devastating. Yet, another crisis, this time provoked by bacteria resistant to existing antibiotics, or 'superbugs', is looming nearby ${ }^{1}$. Antibiotics, long revered as the miracle drug, are rapidly losing effectiveness. The mortality and economic burden related to current antibiotic resistance and future projections are alarming ${ }^{2}$. Although antibiotic resistance occurs naturally, its emergence and spread have been greatly accelerated owing to the inappropriate and excessive use of antibiotics in the past few decades. The expansion of antibiotic use in agriculture and animal farming further exacerbates the situation. The crisis is also compounded by the retreat of the biopharmaceutical industry from developing new antibiotics owing to the lack of incentives ${ }^{3}$. Without effective medicine, we will return to the pre-antibiotic era and face the reality that bacterial infections once easy to treat become untreatable ${ }^{4}$.

Traditional antibiotics are primarily small molecules designed to inhibit bacterial synthesis of DNA, RNA, protein and the cell wall. In parallel, bacteria exploit an array of mechanisms to acquire resistance ${ }^{5}$. The pathogens can render their cell wall structure impermeable to drug molecules, overexpress multidrug efflux pumps to expel drugs, reshuffle the genetic code of drug targets to reduce the susceptibility, or secrete offensive enzymes to destroy drugs before they reach their targets. Bacteria mutate incessantly with each mechanism continually evolving. By contrast, the mechanisms of action of antibiotics are likely preset and unvarying, and thus the efficacy of antibiotics is wavering. When confronted with such an imbalance between the evolution of bacteria and antibiotics, researchers turn to nanomaterials for solutions ${ }^{6}$. The nanometer-scale of these materials allows not one, but a large number of molecules to assemble for dynamic interfacing with both the host and the pathogen. Owing to this attribute, nanomaterials have been quickly developed as standalone 'nano-objects' that enable new therapeutics to arise. Bulk materials with nanostructured surfaces have also been made to prevent bacterial attachment or to kill attached bacteria. Nanomaterials are promising in reducing antibiotic use for infections associated with medical devices and implants. In both areas, nanomaterials leverage distinct mechanisms against bacterial infections (FIG. 1).

In an article in Nature Reviews Microbiology, Rotello and co-authors review the bactericidal mechanisms of various standalone nanomaterials, such as nanoparticles, nanorods, nanowires and $2 \mathrm{D}$ materials ${ }^{7}$. The mechanisms can be generally divided into two categories: augmenting the potency of existing antibiotics and exerting entirely new bactericidal actions without antibiotics. In the first category, nanomaterials act as carriers, and their design is primarily focused on orchestrating the tempo-spatial release of the antibiotic payload. For example, nanomaterials can preferentially deliver antibiotics to the with continued research and development, nanomaterials may become a mainstay for treating bacterial infections in the era of antibiotic resistance

bacteria, a mechanism that enhances drug potency by reducing overall drug exposure. These nanomaterial-based drug carriers can overcome cellular barriers and deliver antibiotics to the cytoplasm to kill intracellular bacteria. Besides, nanomaterials can deliver multiple antibiotics and programme their release profiles to inhibit more than one bacterial target. In the second category, nanomaterials confer entirely new mechanisms that kill bacteria without using antibiotics. These nanomaterials can generate lethal damages to the pathogens through predominantly physical or biochemical processes. Rotello and co-authors also highlight unique challenges presented by biofilms, which protect the bacteria and induce resistance. In this regard, nanomaterials can break extracellular polymeric substances (EPS) through enzymes or mechanical forces. The ability to penetrate deep into the EPS matrix, often achievable by tuning nanomaterial size and surface properties, is essential to enable their bactericidal activities.

In a further article, Ivanova and co-authors focus on nanostructured surfaces and review their antibacterial mechanisms by sieving through both experimental and theoretical studies $^{8}$. In this area, natural antifouling materials, such as plant leaves and insect wings, have been the major source of inspiration. To repel bacteria, a common mechanism is to trap air through superhydrophobicity that reduces the available surface areas for bacteria to attach. To kill the bacteria, one mechanism is to deform and disrupt bacterial cell walls by stretching the bacteria through the attraction from different contact points. Another mechanism is to use sharp, blade-like nanostructures: when the surfaces pull the bacteria close, these structures pierce into the bacteria and create cell-destructive pores. The authors' analysis reveals that effective nanostructures, though initially inspired by nature, are not a mere translation of observation. Instead, they are designed and engineered by first 
understanding the underlying principles that govern the optimized patterns in biological systems and then establishing constraints and boundary conditions associated with fabrication and economic capabilities of the synthetic materials ${ }^{9}$.

Although the two Reviews focus on different branches of antimicrobial nanomaterials, both explicitly emphasize the importance of the relationship between material structures and antibacterial mechanisms. For standalone nano-objects, the clear understanding of this

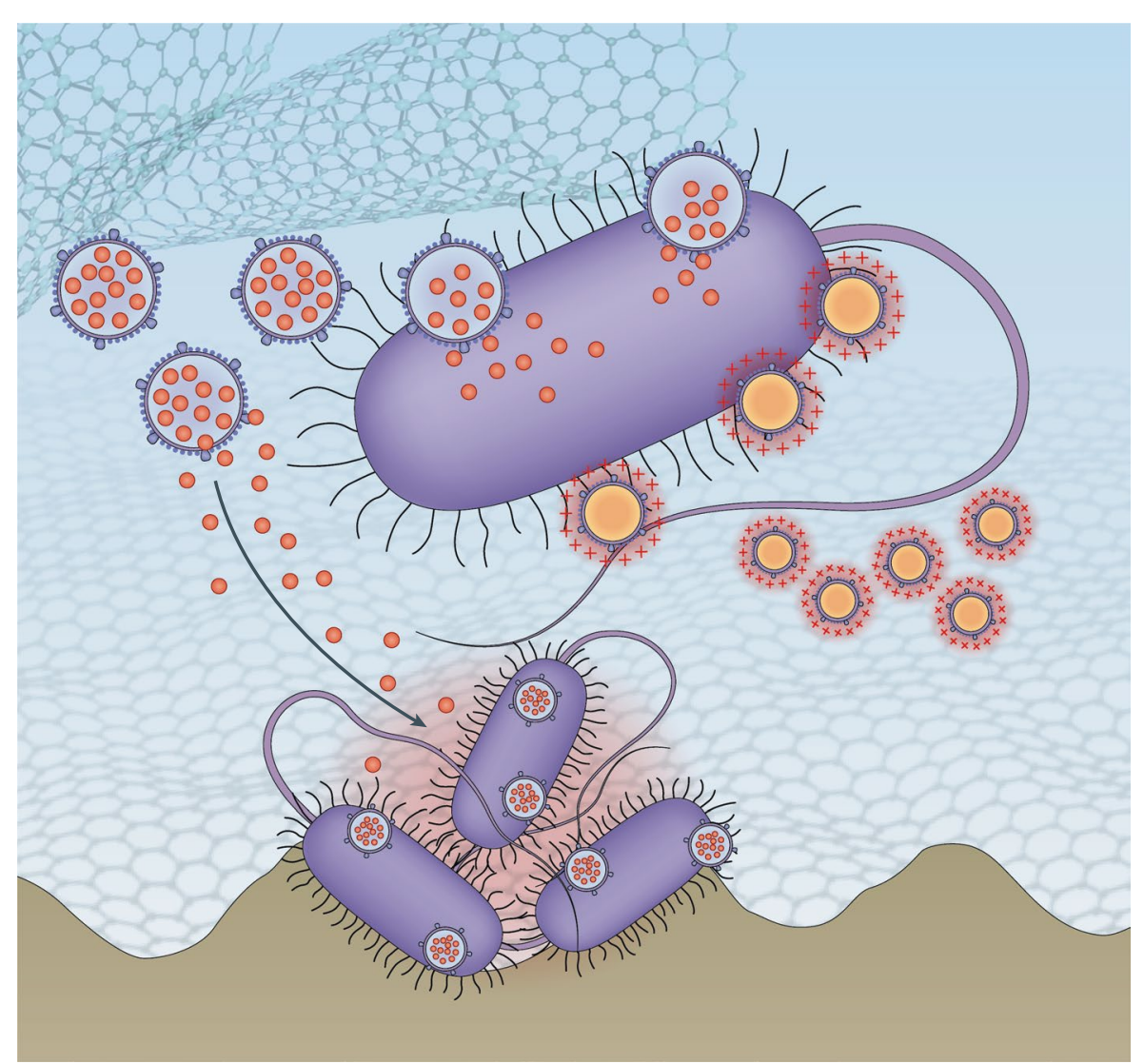

Fig. 1 | Nanomaterials have major potential for treating antibiotic-resistant bacterial infections. One emerging area is standalone 'nano-objects' that either improve the potency of antibiotics or confer bactericidal activity without using antibiotics. Another emerging area is nanostructured surfaces that prevent bacterial attachment or kill those attached bacteria through physico-mechanical interactions with the bacteria.

relationship leads to strategic selection and integration of functional elements. For nanostructured surfaces, the understanding and effective translation of this relationship from nature make a successful 'reconstruction' of natural properties with synthetic materials possible. The structure-mechanism relationship will remain essential for the rational design of the current and future antibacterial nanomaterials. Indeed, elucidating this relationship has had a crucial role in the recent development of biomimetic nanomaterials.
For instance, the emerging cell membranecoated nanoparticle technology integrates natural cell membranes and synthetic nanoparticles to achieve novel functions for the treatment of antibiotic-resistant bacteria ${ }^{10}$. From the perspective of clinical translation, authors from both articles point out major hurdles on the road ahead. The solutions to address these hurdles may also hinge upon in-depth understanding of structuremechanism relationships, which will guide product development with precise assessment and seamless integration of medical needs, manufacturing capabilities and economic constraints. Overall, with continued research and development, nanomaterials may become a mainstay for treating bacterial infections in the era of antibiotic resistance.

Weiwei Gao (iD) and Liangfang Zhang (D) ${ }^{凶}$

Department of NanoEngineering, Chemical Engineering Program, and Moores Cancer Center, University of California San Diego, La Jolla, CA, USA.

凶e-mail: zhang@ucsd.edu

https://doi.org/10.1038/s41579-020-00469-5

1. Lesho, E. P. \& Laguio-Vila, M. The slow-motion catastrophe of antimicrobial resistance and practical interventions for all prescribers. Mayo Clin. Proc. 94, 1040-1047 (2019).

2. Dodds, D. R. Antibiotic resistance: A current epilogue. Biochem. Pharmacol. 134, 139-146 (2017).

3. Nathan, C. Antibiotics at the crossroads. Nature 431, 899-902 (2004)

4. Cox, E., Nambiar, S. \& Baden, L. Needed: antimicrobial development. New Engl. J. Med. 380, 783-785 (2019).

5. Blair J. M. A Webber M. A., Baylay, A. J. Ogbolu, D. O. \& Piddock, L. J. V. Molecular mechanisms of antibiotic resistance. Nat. Rev. Microbiol. 13, 42-51 (2015).

6. Farokhzad, O. C. \& Langer, R. Impact of nanotechnology on drug delivery. ACS Nano 3, 16-20 (2009).

7. Makabenta, J. M. V. et al. Nanomaterial-based therapeutics for antibiotic-resistant bacterial infections. Nat. Rev. Microbiol. https://doi.org/ 10.1038/s41579-020-0420-1 (2020).

8. Linklater, D. P. et al. Mechano-bactericidal actions of nanostructured surfaces. Nat. Rev. Microbiol. https://doi.org/10.1038/s41579-020-0414-z (2020).

9. Aizenberg, J. \& Fratzl, P. Biological and biomimetic materials Adv Mater 21,387-388 (2009).

10. Fang, R. H., Kroll, A. V., Gao, W. \& Zhang, L. Cell membrane coating nanotechnology. Adv. Mater. 30, 1706759 (2018)

Competing interests

The authors declare no competing interests. 